\title{
cDNA microarray analysis assists in diagnosis of malignant intrarenal pheochromocytoma originally masquerading as a renal cell carcinoma
}

\author{
M Takahashi, X J Yang, S McWhinney, N Sano, C Eng, S Kagawa, B T Teh, H-O Kanayama
}

J Med Genet 2005;42:e48 (http://www.jmedgenet.com/cgi/content/full/42/8/e48). doi: 10.1136/jmg.2005.031708

Intrarenal pheochromocytoma (paraganglioma) is a very rare tumour. Its diagnosis is often difficult to establish because of its rarity and its histological similarity to renal cell carcinoma (RCC). Recently, we examined the molecular signatures of different subtypes of kidney tumours by using cDNA microarray. The signature pattern for one tumour, which was originally diagnosed as granular cell RCC, was clearly distinct from that of any other subtype of kidney tumour, and led us to re-evaluate the case. Haematoxylin and eosin staining revealed histological features suggestive of pheochromocytoma, and immunohistochemical studies showed positive staining for neuroendocrine markers but not for keratin. A germline missense mutation, DI 19E, in the familial paraganglioma related gene succinate dehydrogenase subunit $D(S D H D)$, was subsequently identified. The treatment modality was revised and radiotherapy was given, to which the patient responded, leading to a reduction in tumour size of $25 \%$ within the first month. To our knowledge, this is the first report of an intrarenal pheochromocytoma that was diagnosed with the assistance of cDNA microarray analysis.

P heochromocytomas are tumours of neuroectodermal origin arising from chromaffin cells, which produce catecholamines. They are usually derived from the adrenal medulla, but approximately $10 \%$ arise from sympathetic ganglia (extra-adrenal pheochromocytomas or paragangliomas). Pheochromocytomas have been described in several well known hereditary cancer syndromes including multiple endocrine neoplasia type 2, von Hippel-Lindau disease, and neurofibromatosis type 1. Hereditary paragangliomas (PGL) have also been associated with germline mutations of the genes that encode three of the four subunits of mitochondrial complex II, succinate dehydrogenase (SDH) B, C, and D. ${ }^{1}$ When such tumours occur in extra-adrenal sites, their diagnosis can be challenging; for example, to date there are only three reported cases of intrarenal pheochromocytoma. ${ }^{2-4}$

We conducted a study of gene expression profiles of kidney tumours using a cDNA microarray technique. These gene expression profiles can serve as the molecular signatures of particular tumours, and different groups of genes may correlate with the behaviour of the tumours (for example, invasiveness, angiogenesis), clinical outcome, and drug response. ${ }^{5}$ In this report, we describe how, with the help of gene expression profiling, immunohistochemical staining, and mutation analysis, we identified a case of intrarenal pheochromocytoma that originally masqueraded as a renal cell carcinoma.

\section{MATERIALS AND METHODS}

The study was approved by the ethics committee of the University of Tokushima, Japan, and informed written consent was obtained from the patient.

\section{Patient}

A 26 year old man presented with hypertension (180/ $100 \mathrm{mmHg}$ ) in 1997. Pheochromocytoma was investigated, but no conclusive evidence was found. Plasma adrenaline and noradrenaline, cortisol, renin, aldosterone, urinary vanillylmandelic acid and homovanillic acid were within normal limits. Ultrasonography demonstrated a right renal mass. Abdominal computerised tomography revealed a solid mass in the right renal area, measuring approximately $9 \times 5 \mathrm{~cm}$ (fig lA) that contained a low density area indicating necrosis. Marked lymphadenopathy was found from the level of the coeliac arterial trunk to the level of bifurcation of the common iliac artery, with the largest lymph node exceeding $5 \mathrm{~cm}$ in diameter. MRI showed the same intensity as renal cortex in a Tl weighted image and showed high intensity in a T2 weighted image, suggesting necrosis in the tumour. No other lesions were found in the chest, mediastinum, or pelvis. Selective renal arteriography revealed a hypervascular tumour in the middle to lower pole of the right kidney with tumour blush in venous phase (fig 1B).

Pathological examination of a percutaneous biopsy could not confirm the diagnosis. Following embolisation of the right renal artery, a right nephrectomy and lymphadenectomy were performed via a transabdominal approach. The right adrenal gland was not involved. The patient's blood pressure was well controlled during the surgery. Histopathologically, the tumour was diagnosed by haematoxylin and eosin staining to be a renal cell carcinoma (RCC) of the granular cells, with renal capsular penetration and lymph node metastases (fig 2A). Postoperatively, the patient was normotensive and his "RCC" was treated with $5 \times 10^{6}$ units of interferon- $\alpha$, cimetidine, and tegaful uracil. Ten months after the operation, enlarged para-aortic lymph nodes were detected and then resected. The resected lymph node was also histopathologically diagnosed as metastases of granular cell RCC.

\section{cDNA microarray findings}

In 2001, we were analysing the gene expression profiles of seven different histological subtypes of kidney tumours to determine if these subgroups can be distinguished by their gene expression profiles using microarrays containing 19968 cDNAs. ${ }^{6}$ In total, 70 kidney tumours (including 39 clear cell RCCs, one metastatic and seven primary papillary RCCs, six

Abbreviations: AML, acute myeloid leukaemia; MIBG, metaiodobenzylguanidine; RCC, renal cell carcinoma; SDH, succinate dehydrogenase 

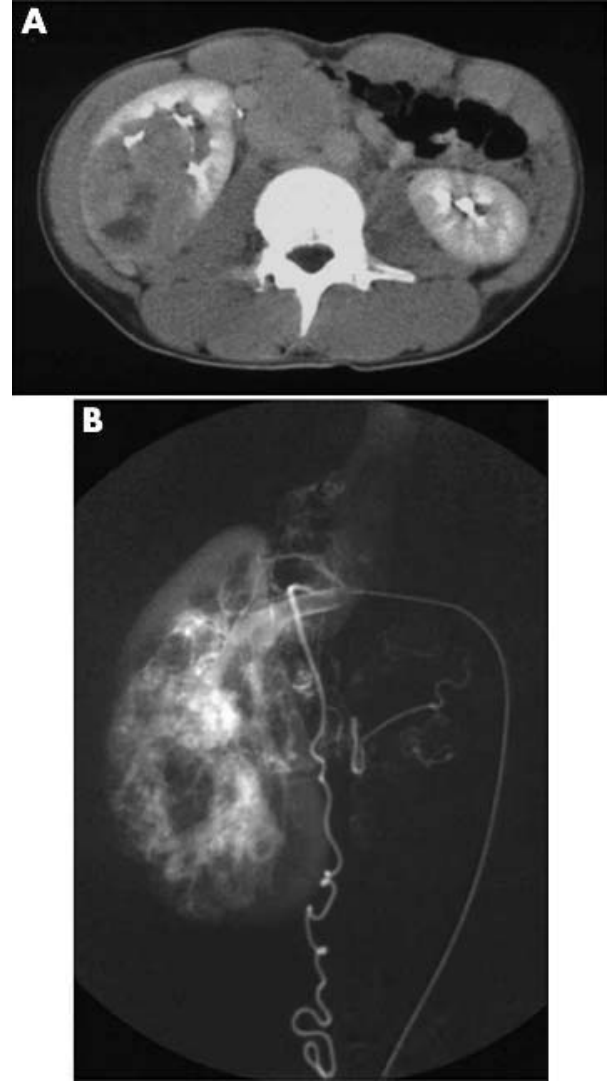

Figure 1 Imaging results. (A) Abdominal computerised tomography revealed a solid mass in the right renal area, approximately $9 \times 5 \mathrm{~cm}$ in size, which contained a low density area indicating necrosis. Marked lymphadenopathy can be seen. (B) Selective renal arteriography with tumour staining revealed a hypervascular tumour in the middle to lower pole of the right kidney and a dilated right renal vein.

granular cell RCCs (including the present case), five chromophobe RCCs, two sarcomatoid RCCs, two oncocytomas, three transitional cell carcinomas (TCCs) of the renal pelvis, and five Wilms tumours) were compared with noncancerous kidney tissues. Based on the expression patterns of 3560 selected cDNAs, we found distinct molecular signatures in the clear cell, papillary, chromophobe RCC/ oncocytoma, TCC, and Wilms subtypes, whereas the six cases with granular cell RCC did not show a common signature (fig 3).
Pathological and immunohistochemical evaluation of "granular cell RCC"

These "granular RCC" cases were further reviewed histologically by a urological pathologist (XJY), blinded to the study. All granular cell RCC cases except the present case were reclassified as clear cell RCC $(n=4)$ or oncocytoma $(n=1)$, which were compatible with gene expression classification. The present case, which was not clustered with any subtype of RCC, was also reevaluated using NSE, HMB45, synaptophysin, S100, and keratin immunochemistry (performed as previously described). ${ }^{7}$

Mutation analysis of succinate dehydrogenase genes The genes encoding the mitochondrial complex II subunits $S D H A, S D H B, S D H C$, and $S D H D$, were screened for mutations as previously reported. ${ }^{8}$ PCR amplification was carried out in $1 \times$ PCR buffer (Qiagen) containing $200 \mu \mathrm{mol} / \mathrm{l}$ deoxynucleotide triphosphate, $0.6 \mu \mathrm{mol} / \mathrm{l}$ of each primer, $2.5 \mathrm{U}$ Taq polymerase (Qiagen), and 100-200 ng of tumour DNA template in a $50 \mu \mathrm{l}$ volume. PCR conditions were one cycle of 15 minutes at $95^{\circ} \mathrm{C}$ then 35 cycles of 1 minute at $95^{\circ} \mathrm{C}$, 1 minute at $58{ }^{\circ} \mathrm{C}$, and 1 minute at $72^{\circ} \mathrm{C}$, which was followed by one cycle of 10 minutes at $72^{\circ} \mathrm{C}$.

PCR amplicons were purified by gel electrophoresis (BioRad Laboratories, Hercules, CA) and column purification (Wizard PCR Prep; Promega, Madison, WI), and subjected to semiautomated sequencing using the aforementioned primers with a dye terminator method, and sequenced on an ABI377xl or PE3700 sequencer. The sequencing was repeated on DNA of the matched normal kidney.

\section{RESULTS}

Histology showed clusters of tumour cells with granular cytoplasm and prominent vascularity, suggestive of pheochromocytoma, extensively infiltrating the kidney parenchyma (fig 2A, B). Further immunohistochemical analysis showed positive staining for NSE, HMB45, and synaptophysin (focally positive) and negative staining for keratin in cancer cells, and positive staining for S100 in sustentacular cells (fig 2-E). These findings further support the diagnosis of pheochromocytoma or paraganglioma. Interestingly, we also identified a germline mutation, D119E, in the SDHD gene (fig 4). Two common polymorphisms were also found, T891C in SDHA and Cl8A in SDHB.

Five years after the nephrectomy, the patient is alive with continuing disease. He had an enlarging para-aortic mass without hypertension or elevation of any catecholamine related markers in blood and urine. Strong adhesion around the duodenum and inferior vena cava prevented further surgical resection of the residual tumour. After a diagnosis of
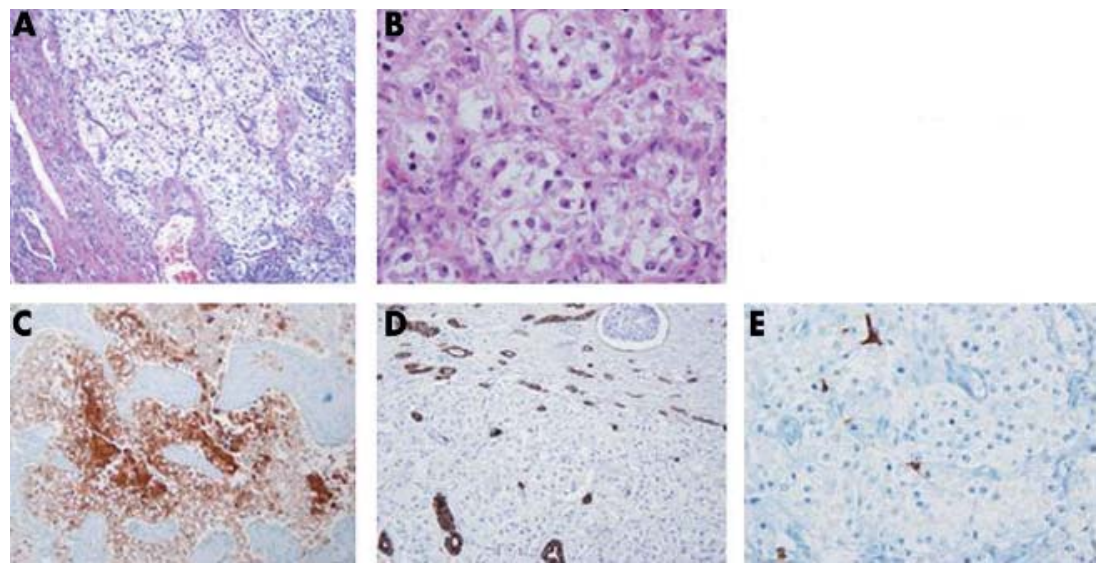

Figure 2 Intrarenal pheochromocytoma. (A) Low magnification section showing tumour cells (right side) infiltrating renal cortex (left low corner) (haematoxylin and eosin). (B) High magnification section showing tumour cells with fine granular cytoplasm and rich microvessels (haematoxylin and eosin). (C) Positively stained tumour cells (NSE immunohistochemistry). (D) Nonreactivity in tumour cells but reactivity in residual renal tubules (keratin immunohistochemistry; AEl / AE3). (E) Positively stained sustentacular cells (S-100 immunohistochemicstry). 


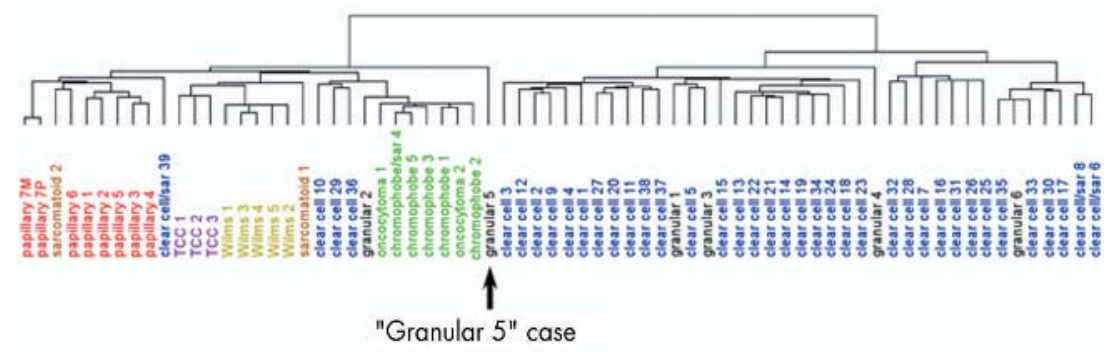

Figure 3 Clustering of 70 kidney tumours. The clustering of patients (using Pearson's correlation) is based on global gene expression profiles. The tumours clustered into two broad groups with one group consisting of primarily clear cell RCC and the other consisting of all other kidney tumours. Five chromophobe RCCs and two oncocytomas are clustered close together. Eight papillary RCCs, five Wilms tumours, and three TCC are clustered together as groups. The case "granular 5," which has its own distinct molecular signature, does not cluster with any other type of kidney tumous.

malignant intrarenal pheochromocytoma was established, the patient underwent external beam radiation therapy, delivering $50 \mathrm{~Gy}$ to the residual para-aortic tumours. One month after radiation therapy, MRI revealed a $25 \%$ reduction in the para-aortic masses.

\section{DISCUSSION}

Previously, in a microarray study of acute leukaemia, one particular case showed a gene expression profile that fitted neither acute myeloid leukaemia (AML) nor acute lymphoblastic leukaemia. ${ }^{9}$ This brought into question the diagnosis of that case as AML. Subsequent analysis found that the tumour had high expression of certain particular genes suggesting a mesenchymal origin. Further cytogenetic study, which revealed a pathognomonic translocation, confirmed it as an alveolar rhabdomyosarcoma, and the treatment for the patient was revised accordingly.

Similarly, our microarray based molecular classification study of RCC revealed that a previous diagnosis of RCC was actually a rare malignant intrarenal pheochromocytoma. This change in diagnosis was only possible because of the gene expression microarray. The molecular profile of this case clearly did not fit with any of the other RCC cases, leading to further investigation by immunohistochemical staining and mutation analysis. Using normal kidney tissues as a control allowed us to find a marked difference in the molecular signature of this tumour. Ideally, we should have obtained the tumour's own molecular signature compared to normal adrenal tissues, but we did not have enough RNA for obtaining particularly altered gene expressions. In addition, we found that this patient carries a germline missense mutation, D119E, in exon 4 of SDHD. This amino acid, and those surrounding it, is highly conserved down to mouse and rat. The D119E mutation is predicted to disrupt assembly of complex II. ${ }^{10}$ The patient does not have a family history of hereditary paraganglioma and the mutation could well represent a de novo mutation. Unfortunately, neither parent was available for mutation analysis.

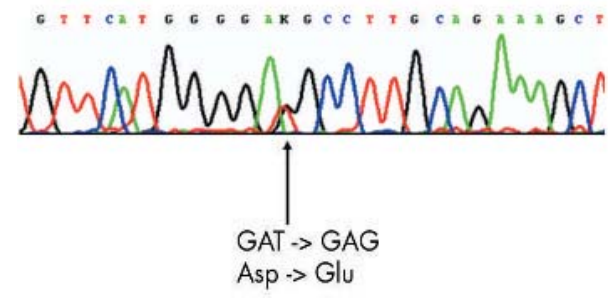

Figure 4 Germline D1 19E (exon 4) mutation; the succinate dehydrogenase subunit $D(S D H D)$ gene.
Because of further immunohistochemical and molecular studies, the treatment modality for this patient was revised, a change to which the patient has responded positively. The diagnosis of malignant pheochromocytoma (paraganglioma) instead of RCC had a great effect on the treatment approach. Interferon and interleukin-2 are still the mainstay treatment for metastatic RCC, and radiation therapy is generally considered ineffective except for palliation in bone metastasis or stereotactic radiosurgery for brain metastasis. ${ }^{11}{ }^{12}$ In contrast, there are several reports on the response of malignant pheochromocytoma to radiation therapy, including external beam radiotherapy or radiolabelled metaiodobenzylguanidine (MIBG). ${ }^{13-15}$ As MIBG scintigraphy revealed no accumulation of MIBG in the tumour, radiolabelled MIBG therapy was not indicated in this case. Combination chemotherapy of cyclophosphamide, vincristine, and dacarbazine or of these three agents with doxorubicin have also been reported as effective in treating malignant pheochromocytoma. ${ }^{15-17}$ After providing these treatment options to the patient, he decided to undergo external beam radiotherapy for his para-aortic tumours. He has experienced a partial response ( $25 \%$ reduction in size to the radiotherapy), and continues under follow up.

\section{CONCLUSION}

We report a very rare case of intrarenal pheochromocytoma in a 26 year old man whose final diagnosis was first suspected because of microarray gene expression profiling. This then led to further immunohistochemical staining for neuroendocrine markers and mutation analysis of the known paraganglioma associated genes, which revealed an unexpected predisposing germline mutation. This case illustrates the importance and clinical implications of microarray expression profiling.

\section{ACKNOWLEDGEMENTS}

This work was supported by the Fischer Family Trust Foundation, the Hauenstein Family Foundation, and the Van Andel Research Institute. We would like to thank $\mathrm{S}$ Antio for assistance with manuscript preparation and submission.

\footnotetext{
Authors' affiliations

M Takahashi, S Kagawa, H-o Kanayama, Department of Urology, The University of Tokushima, School of Medicine, 3-18-15 Kuramoto-cho, Tokushima 770-8503, Japan

X J Yang, Departments of Pathology and Surgery/Urology, University of Chicago, 5841 South Maryland Avenue, Chicago, IL 60637, USA S McWhinney, Human Cancer Genetics Program, Comprehensive Cancer Center, Department of Molecular Genetics, The Ohio State University, Columbus, OH 43210, USA

N Sano, Department of Pathology, The University of Tokushima, School of Medicine, 3-18-15 Kuramoto-cho, Tokushima 770-8503, Japan
} 
C Eng, Clinical Cancer Genetics Program, Comprehensive Cancer Center, Division of Human Genetics, Department of Internal Medicine and Department of Molecular Genetics, The Ohio State University, Columbus, $\mathrm{OH} 43210$, USA

B T Teh, Laboratory of Cancer Genetics, Van Andel Research Institute, 333 Bostwick Avenue, N.E., Grand Rapids, MI 49503, USA

Competing interests: none declared

Correspondence to: Dr B T Teh, Laboratory of Cancer Genetics, Van Andel Research Institute, 333 Bostwick Avenue NE, Grand Rapids, MI 49503, USA; bin.teh@vai.org

Received 4 February 2005

Revised 22 March 2005

Accepted 24 March 2005

\section{REFERENCES}

1 Baysal BE, Ferrell RE, Willett-Brozick JE, Lawrence EC, Myssiorek D, Bosch A, van der Mey A, Taschner PE, Rubinstein WS, Myers EN, Richard CW, III, Cornelisse CJ, Devilee P, Devlin B. Mutations in SDHD, a mitochondrial complex II gene, in hereditary paraganglioma. Science 2000;287:848-51.

2 Simon H, Carlson DH, Hanelin J, Kleeman F, Feen D. Intrarenal pheochromocytoma: report of a case. J Urol 1979;121:805-7.

3 Bezirdjian DR, Tegtmeyer CJ, Leef JL. Intrarenal pheochromocytoma and renal artery stenosis. Urol Radiol 1981;3:121-2.

4 Rothwell DL, Vorstman B, Patton I, Allan JS. Intrarenal pheochromocytoma. Urology 1983;21:175-7.

5 Ladanyi M, Gerald WL. Expression profiling of human tumors: diagnostic and research applications. New Jersey: Humana Press, 2003:399.
6 Takahashi M, Yang XJ, Sugimura J, Backdahl J, Tretiakova M, Qian C, et al. Molecular sub-classification of kidney cancer and the discovery of new diagnostic markers. Oncogene 2003;22:6810-18.

7 Tretiakova MS, Sahoo S, Takahashi M, Turkyilmaz M, Vogelzang N, Lin F, Krausz T, Teh BT, Yang XJ. Expression of alpha-methylacyl-CoA racemase in papillary renal cell carcinoma. Am J Surg Pathol 2004;28:69-76.

8 McWhinney SR, Pilarski R, Forrester S, Schneider M, Sarquis MM, Dias EP Eng C. Large germline deletions of mitochondrial complex II subunits $S D H B$ and SDHD in hereditary paraganglioma. J Clin Endocrinol Metab 2004:89:5694-9.

9 Golub TR, Slonim DK, Tamayo P, Huard C, Gaasenbeek M, Mesirov JP, et al. Molecular classification of cancer: class discovery and class prediction by gene expression monitoring. Science 1999;286:531-7.

10 Eng C, Kiuru M, Fernandex MJ, Aaltonen LA. A role for mitochondrial enzymes in inherited neoplasia and beyond. Nat Rev Cancer 2003;3:193-202.

11 Cutuli BF, Methlin A, Teissier E, Schumacher C, Jung GM. Radiation therapy in the treatment of metastatic renal-cell carcinoma. Prog Clin Biol Res 1990;348:179-86.

12 Sheehan JP, Sun MH, Kondziolka D, Flickinger J, Lunsford LD. Radiosurgery in patients with renal cell carcinoma metastasis to the brain: long-term outcomes and prognostic factors influencing survival and local tumor control. $J$ Neurosurg 2003;98:342-9.

13 Yu L, Fleckman AM, Chadha M, Sacks E, Levetan C, Vikram B. Radiation therapy of metastatic pheochromocytoma: case report and review of the literature. Am J Clin Oncol 1996;19:389-93.

14 Sisson JC. Radiopharmaceutical treatment of pheochromocytomas. Ann N Y Acad Sci 2002;970:54-60.

15 Nakabeppu Y, Nakajo M. Radionuclide therapy of malignant pheochromocytoma with 131I-MIBG. Ann Nud Med 1994;8:259-68.

16 Sisson JC, Shapiro B, Shulkin BL, Urba S, Zempel S, Spaulding S. Treatment of malignant pheochromocytomas with 131-I metaiodobenzylguanidine and chemotherapy. Am J Clin Oncol 1999;22:364-70.

17 Tato A, Orte L, Diz P, Quereda C, Ortuno J. Malignant pheochromocytoma, still a therapeutic challenge. Am J Hypertens 1997;10:479-81. 